\title{
96-Well Plasmonic Sensing with Nanohole Arrays
}

Maxime Couture ${ }^{1}$, Korak Kumar Ray ${ }^{1}$, Hugo-Pierre Poirier-Richard ${ }^{1}$, Anthony Crofton ${ }^{1}$ and Jean-Francois Masson*1,2

${ }^{1}$ Département de chimie, Université de Montréal, CP. 6128 Succ. Centre-Ville, Montréal, Qc, Canada, H3C $3 J 7$

${ }^{2}$ Centre for self-assembled chemical structures (CSACS)

* Corresponding author: jf.masson@umontreal.ca ; tel : +1-514-343-7342

\section{Supporting information}

Figures SI1 to SI3 are provided as supporting information. These figures reports technical details regarding the software and design of the 96 -well plate reader. 


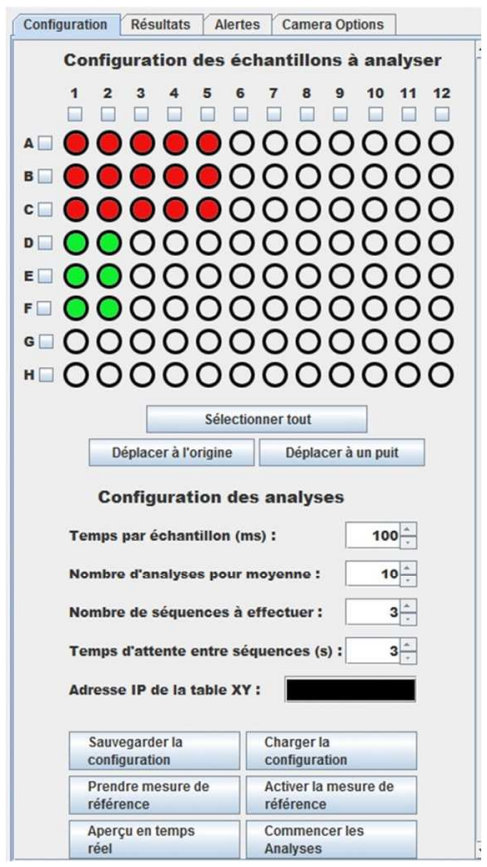

Figure SI1. Software interface for the control of the multi-well plate reader and camera parameters.

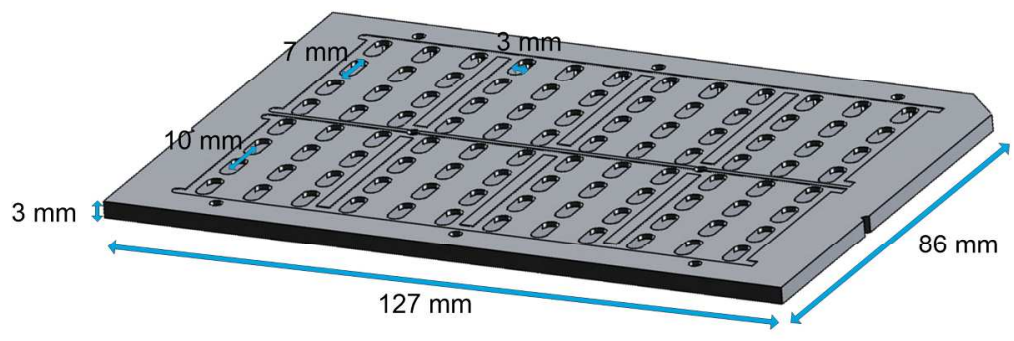

Figure SI2. Scheme of the bottom aluminium part of the multi-well plate with dimension. 


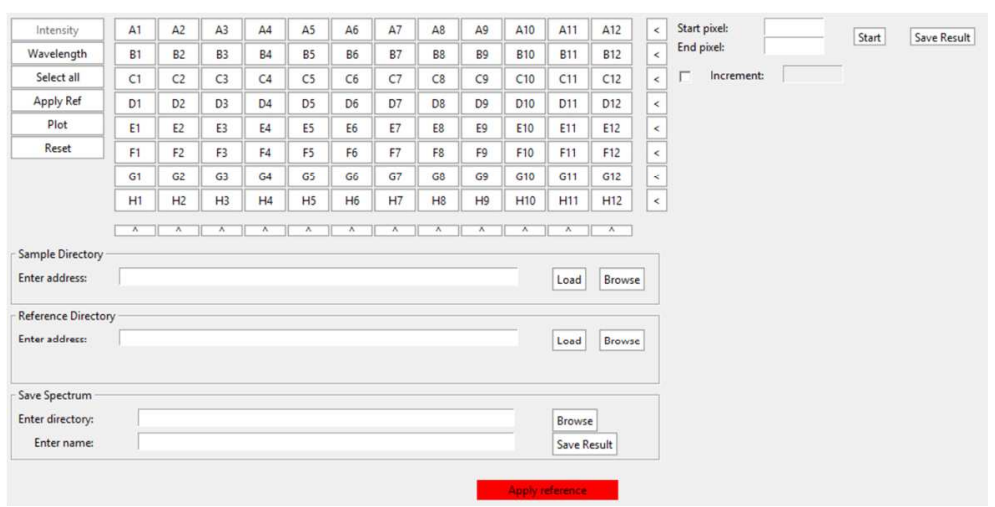

Figure SI3. Software interface for the data treatment. 